\title{
Development and Validation of a Nomogram Based on CA125 Levels for Predicting Progression-free Survival in Patients with Ovarian Cancer
}

Minjun He ( $\sim$ hemj1@sysucc.org.cn )

Sun Yat-sen University Affiliated Tumor Hospital: Sun Yat-sen University Cancer Center https://orcid.org/0000-0001-8375-7663

Hongyu Peng

Sun Yat-sen University Cancer Center

Zhifeng Liu

Guangdong Provincial People's Hospital

\section{Quanyang Gao}

The Fifth Affiliated Hospital of Guangzhou Medical University

\section{Research}

Keywords: Nomogram, Ovarian cancer, CA125 levels, Progression-free Survival

Posted Date: January 11th, 2021

DOl: https://doi.org/10.21203/rs.3.rs-35294/v2

License: (a) (i) This work is licensed under a Creative Commons Attribution 4.0 International License. Read Full License 


\section{Abstract}

Background Ovarian cancer is the leading cause of death in patients with gynecological cancers, and it has very low survival. CA125 have been shown to be an important prognostic factor for PFS, Therefore, we aimed to develop and validate a nomogram incorporating CA125 levels after three cycles of chemotherapy for predicting progression-free survival (PFS) in patients with ovarian cancer.

Methods The nomogram was developed in a primary cohort of 491 patients with stage II-IV ovarian cancer. Performance was assessed by concordance index (C-index), calibration curve, and decision curve analysis, and compared with the International Federation of Gynecology and Obstetrics (FIGO) staging system. The predictive value of CA125 levels after three cycles of chemotherapy was evaluated. The model was subjected to bootstrap internal validation. An independent cohort of 81 patients was used for external validation.

Results CA125 levels after three cycles of chemotherapy were significantly associated with PFS. Five variables, including CA125 levels were selected to develop the nomogram. The nomogram demonstrated adequate discrimination, with a bootstrap-corrected C-index of 0.708 , and good calibration. External validation of the nomogram achieved excellent discrimination (C-index, 0.724) and calibration. CA125 levels after three adjuvant chemotherapy cycles showed a marginally significant increment of discrimination to the nomogram in the primary cohort (C-index, 0.708 vs $0.668 ; P=0.097$ ). Superior discriminative ability was observed in the nomogram when compared with the FIGO staging system only in the primary cohort (C-index, 0.708 vs $0.578 ; \mathrm{P}<0.001$ ). Decision curve analysis demonstrated that our nomogram was clinically useful.

Conclusion We developed and validated a nomogram incorporating CA125 levels after three chemotherapy cycles for PFS prediction in ovarian cancer. This nomogram showed well-predictive performance and easy clinical application.

\section{Introduction}

Ovarian cancer is the leading cause of death in patients with gynecologic malignancies. In 2018, about 22,240 new cases of ovarian cancer and 14,070 deaths from the disease are projected to occur in the United States.[1] In 2015, there were approximately 52,100 new cases of ovarian cancer and 22,500 deaths in China.[2] Most women are diagnosed at an advanced stage, with a 5-year overall survival (OS) rate lower than $25 \% .[3,4]$ Therefore, accurate prediction of patient outcome plays an important role in determining treatment strategies.

Nomograms are widely used as predictive tools in many cancers. They are able to provide accurate risk estimates tailored to the individual patient, which facilitates patient counseling and treatment selection. [5] Several nomograms have been developed for ovarian cancer prognostic prediction.[6-8] However, none incorporated the change in cancer antigen 125 (CA125) levels following treatment, which may have great potential in improving the predictive accuracy. 
CA125 is encoded by the MUC16 gene in humans, and has been investigated extensively as a tumor marker in ovarian cancer.[9-11] A series of studies have demonstrated that the normalization of CA125 levels at different time-points (e.g. the first,[11] second,[12] third,[13-15] and sixth[16] chemotherapy cycles) is significantly associated with the prognosis of patients with ovarian cancer. Lee et al. found that CA125 levels $\leq 35 \mathrm{U} / \mathrm{mL}$ after the first cycle of chemotherapy and the normalization of CA125 levels within three cycles of chemotherapy were two of the most significant prognostic factors for both overall survival (OS) and progression-free survival (PFS) in patients with ovarian cancer.[11] Consistent with these observations, we found that the normalization of CA125 levels after three cycles of chemotherapy was an independent prognostic factor of improved survival for advanced ovarian cancer in our previous study. [14]

In this study, we aimed to develop a nomogram for the prediction of PFS in ovarian cancer incorporating CA125 levels after three cycles of chemotherapy, along with other prognostic factors. We expect that the integration of CA125 levels after three cycles of chemotherapy could improve the predictive accuracy of the nomogram for ovarian cancer.

\section{Methods}

\section{Patients}

Ethical approval was obtained from the institutional review board of Sun Yat-sen University Cancer Centre. The need for informed consent was waived since no identifiable information was used in this study.

An evaluation of the institutional database for medical records from January 1994 to December 2014 was performed to identify patients with ovarian cancer who received initial treatment at the Sun Yat-sen University Cancer Centre. Only patients who met the following criteria were included in the study: newly diagnosed, histologically confirmed epithelial ovarian cancer; stage II-IV disease; serially measured CA125 levels; and complete follow-up data. In total, 1740 patients were identified; 491 patients who met the above-mentioned inclusion criteria were included as the primary cohort (the training cohort).

The clinicopathologic data were extracted, including age, neutrophil-to-lymphocyte ratio, platelet count, serum albumin level, International Federation of Gynecology and Obstetrics (FIGO) stage, histologic type, tumor grade, diameter of residual disease, CA125 levels after three cycles of chemotherapy (normal CA125 levels were defined as being $\leq 35 \mathrm{U} / \mathrm{mL}$ ), follow-up, recurrence and vital status.

The standard treatment for advanced ovarian cancer in our institution comprised comprehensive staging or primary debulking surgery followed by adjuvant chemotherapy. Neoadjuvant chemotherapy followed by interval debulking surgery was performed in patients with bulky stage III/IV disease who were not eligible surgical candidates. Surgery mainly included total hysterectomy, bilateral salpingo-oophorectomy, omentectomy, lymphadenectomy, and resection of all visible bulky tumor. Bowel resection, splenectomy, and partial liver resection were performed as warranted to remove all macroscopic tumor. Residual 
disease $<1 \mathrm{~cm}$ in maximum diameter after surgery was defined as optimal debulking, whereas residual disease $\geq 1 \mathrm{~cm}$ was considered suboptimal debulking. Adjuvant chemotherapy mainly included $\geq 6$ cycles of platinum-based systemic chemotherapy.

Patients were followed up periodically after primary treatment. Monitoring comprised pelvic examination, abdominal and pelvic ultrasonography, and examination of the levels of CA125 and other tumor markers every 3 months for 3 years, then every 6 months for 2 years, and annually after 5 years. Computed tomography (CT), magnetic resonance imaging (MRI), or positron emission tomography- computed tomography (PET-CT) were performed if clinically indicated.

All procedures were carried out in accordance with the approved guidelines of our institute.

An external validation cohort was screened from patients with ovarian cancer who received initial treatment at other hospitals between January 2001 and August 2016 using the same criteria as the primary cohort. Most of these patients were transferred to our institution because of disease recurrence, and the rest who did not experience recurrence came to our institution for follow-up. It should be noted that only the medical records of initial treatment of these patients at other hospitals were used in this study, which guaranteed these patients as an independent validation cohort. However, the vital status for the external validation cohort was ascertained by reviewing their final medical records at our institution.

\section{Construction of the Nomogram}

PFS was defined as the interval between the date of diagnosis and the date of first recurrence or death, or the date of last contact for living patients without recurrent disease. In the primary cohort, PFS according to different variables was estimated using the Kaplan-Meier method and compared using the log-rank test. Multivariate Cox proportional hazards regression analysis was used to identify the independent prognostic factors. We established the nomogram in the primary cohort (training cohort) by incorporating the significant prognostic factors into the Cox regression model using the "rms" package in R software.

\section{Performance of the Nomogram in the Primary Cohort}

The Harrell's concordance index (C-index) was calculated to estimate the discrimination ability of the nomogram. The nomogram was subjected to 1000 bootstrap resamples for internal validation. The value of the C-index ranges from 0.5 to 1.0 , with 0.5 indicating random chance and 1.0 indicating perfectly corrected discrimination. Calibration plots, which showed the consistency between the outcomes predicted by the nomogram and actual outcomes, were used to assess the robustness of the model.

\section{External Validation of the Nomogram}

The nomogram was applied to the external validation cohort for external validation. Using the same methods as in the primary cohort, discrimination and model calibration were tested.

Incremental Predictive Value of CA125 Levels to the Nomogram 
We investigated the incremental predictive value of the normalization of CA125 levels after three cycles of chemotherapy to the nomogram by comparing the respective $\mathrm{C}$-index for the model with and without CA125 levels after three cycles of chemotherapy.

Comparison of the Nomogram with the FIGO Staging System in Discrimination Ability

Furthermore, we attempted to demonstrate the superiority in discrimination ability of the established nomogram over the FIGO staging system. First, the nomogram and the FIGO staging system were compared with respect to the C-index. Next, patients in the primary cohort were classified into low, moderate, and high risk groups by ranking their nomogram calculated scores into tertiles. The distributions of the PFS for these risk groups, as well as for different FIGO stages, were depicted by the Kaplan-Meier method and analyzed by the log-rank test.

Clinical Use

Decision curve analysis (DCA) was performed to examine the clinical application of the nomogram by quantifying the net benefits at different threshold probabilities in the primary cohort.[17, 18] In addition, DCA was also conducted for the model that did not integrate CA125 levels after three cycles of chemotherapy.

\section{Statistical Analysis}

Statistical analysis was performed using R version 3.5.1 and SAS version 9.3 (SAS Institute Inc. Cary, NC, USA). $P$ values less than 0.05 were considered statistically significant. All tests were two-sided. Details of $\mathrm{R}$ code and SAS codes for running nomograms were shown in Appendix 1.

The key raw data have been recorded at Sun Yat-sen University Cancer Center for future reference (number RDDA 2019000972).

\section{Results}

Clinicopathologic Characteristics of Patients

The clinicopathological characteristics of the primary cohort and external validation cohort are summarized in Table 1. In total, 491 patients were included in the primary cohort; $71.3 \%$ showed normalization of CA125 levels after three cycles of chemotherapy. The median PFS in the primary cohort was 22 months (range 1.0 to 234.3 months). The external validation cohort consisted of 81 patients; $69.1 \%$ showed normalization of CA125 levels after three cycles of chemotherapy. The median PFS in the external validation cohort was 15.0 months (range 3.6 to 61.8 months). Most of the clinical characteristics between the primary and external validation cohorts were comparable. 
Table 1

Patients' clinicopathologic characteristics in the study.

\begin{tabular}{|c|c|c|}
\hline Variable & $\begin{array}{l}\text { Training (Primary) } \\
\text { cohort } \\
(n=491)\end{array}$ & $\begin{array}{l}\text { External validation } \\
\text { cohort } \\
(n=81)\end{array}$ \\
\hline Age (year) & $52( \pm 9.8)$ & $50( \pm 8.4)$ \\
\hline \multicolumn{3}{|c|}{ Examination prior to treatment, Median (IQR) } \\
\hline NLR & $3.1(2.1,4.5)$ & \\
\hline Platelet count $\left(\times 10^{9}\right)$ & $306(229,380)$ & \\
\hline Serum albumin levels in $\mathrm{g} / \mathrm{L}$ & $41(37,44)$ & \\
\hline \multicolumn{3}{|l|}{ FIGO stage, $\mathrm{n}(\%)$} \\
\hline II & $58(11.8)$ & $11(13.6)$ \\
\hline III & $351(71.5)$ & $60(74.1)$ \\
\hline IV & $82(16.7)$ & $10(12.4)$ \\
\hline \multicolumn{3}{|l|}{ Histology, n (\%) } \\
\hline Serous & $408(83.1)$ & $67(82.7)$ \\
\hline Non-Serous & $83(16.9)$ & $14(17.3)$ \\
\hline \multicolumn{3}{|l|}{ Grade, n (\%) } \\
\hline Grade 1 & $20(4.1)$ & $7(8.6)$ \\
\hline Grade 2 & $70(14.3)$ & $15(18.5)$ \\
\hline Grade 3 & $401(81.7)$ & $59(72.8)$ \\
\hline \multicolumn{3}{|l|}{ Residual disease, n (\%) } \\
\hline < 1 cm (Optimal debulking) & $369(75.2)$ & $58(71.6)$ \\
\hline$\geq 1 \mathrm{~cm}$ (Suboptimal debulking) & $122(24.9)$ & $23(28.4)$ \\
\hline \multicolumn{3}{|c|}{$\begin{array}{l}\text { Normalization of CA125 levels after three cycles of } \\
\text { chemotherapy, } n(\%)\end{array}$} \\
\hline Yes & $350(71.3)$ & $56(69.1)$ \\
\hline
\end{tabular}

IQR interquartile range, NLR neutrophil to lymphocyte ratio, $C A 125$ cancer antigen 125

\section{Development and Validation of a Nomogram Based on CA125 Levels for Predicting Progression-free} Survival in Patients with Ovarian Cancer

Minjun $\mathrm{He}^{1,2^{*}}$, Hongyu Peng ${ }^{1,2}$, Zhifeng Liu ${ }^{3}$, Quanyang $\mathrm{Gao}^{4}$ 


\begin{tabular}{|lll|}
\hline Variable & $\begin{array}{l}\text { Training (Primary) } \\
\text { cohort } \\
(\mathbf{n = 4 9 1 )}\end{array}$ & $\begin{array}{l}\text { External validation } \\
\text { cohort } \\
(\mathbf{n}=\mathbf{8 1})\end{array}$ \\
\hline No & $141(28.7)$ & $25(30.9)$ \\
\hline IQR interquartile range, NLR neutrophil to lymphocyte ratio, $C A 125$ cancer antigen 125 \\
\hline $\begin{array}{l}\text { Development and Validation of a Nomogram Based on CA125 Levels for Predicting Progression-free } \\
\text { Survival in Patients with Ovarian Cancer }\end{array}$ \\
\hline Minjun He ${ }^{1,2^{\star}}$, Hongyu Peng $^{1,2}$, Zhifeng Liu ${ }^{3}$, Quanyang Gao \\
\hline
\end{tabular}

Independent Prognostic Factors in the Primary Cohort

The associations between social-demographic, clinical factors and PFS are shown in Table 2. Younger age ( $\leq 50$ vs $₫ 50 ; P=0.010$ ), earlier stage (stage II vs stage III vs stage IV; $P<0.001$ ), and optimal debulking (optimal vs suboptimal; $\mathrm{P}<0.001$ ) were associated with better PFS. Patients with normalization of CA125 levels after three cycles of chemotherapy had superior PFS than patients who did not have normalized CA125 levels $(P<0.001)$. Furthermore, we found that serum albumin levels prior to treatment were a significant prognostic factor $(P<0.001)$. Age $(P=0.019)$, FIGO stage $(P<0.001)$, residual disease $(P<0.001)$, and the normalization of CA125 levels after three cycles of chemotherapy $(P<0.001)$ were identified as independent prognostic factors for PFS (Table 2), and they were entered into the final multivariate Cox regression. 
Table 2

Univariate Analysis and Multivaiate Cox Regression Analysis for the Primary Cohort

\begin{tabular}{|c|c|c|c|c|}
\hline \multirow[t]{2}{*}{ Variable } & \multicolumn{2}{|l|}{ Univariate } & \multicolumn{2}{|l|}{ Multivariate* } \\
\hline & $\mathrm{HR}(95 \% \mathrm{Cl})$ & $P$ value & $\mathrm{HR}(95 \% \mathrm{Cl})$ & $P$ value \\
\hline \multicolumn{5}{|l|}{ Age groups } \\
\hline$\leq 50$ years & Reference & & Reference & \\
\hline$\nabla 50$ years & $1.3(1.1-1.6)$ & 0.010 & $1.3(1.0-1.7)$ & 0.019 \\
\hline NLR in number & $\begin{array}{l}1.02(0.99- \\
1.05)\end{array}$ & 0.228 & $1.02(0.98-1.05)$ & 0.357 \\
\hline Platelet count in number & $\begin{array}{l}1.00(1.00- \\
1.00)\end{array}$ & 0.766 & $1.00(1.00-1.00)$ & 0.778 \\
\hline Serum albumin levels in $\mathrm{g} / \mathrm{L}$ & $\begin{array}{l}0.96(0.94- \\
0.97)\end{array}$ & $<0.001$ & $0.97(0.95-0.99)$ & 0.003 \\
\hline \multicolumn{5}{|l|}{ FIGO stage } \\
\hline II & Reference & & Reference & \\
\hline III & $2.8(1.8-4.3)$ & $<0.001$ & $2.06(1.30-3.27)$ & 0.002 \\
\hline IV & $4.2(2.6-6.7)$ & $<0.001$ & $2.96(1.77-4.95)$ & $<0.001$ \\
\hline \multicolumn{5}{|l|}{ Histology } \\
\hline Serous & Reference & & Reference & \\
\hline Non-Serous & $1.0(0.7-1.3)$ & 0.905 & $1.35(0.97-1.88)$ & 0.076 \\
\hline \multicolumn{5}{|l|}{ Grade } \\
\hline Grade 1 & Reference & & Reference & \\
\hline Grade 2 & $1.0(0.6-1.9)$ & 0.941 & $0.75(0.40-1.43)$ & 0.387 \\
\hline Grade 3 & $1.4(0.8-2.4)$ & 0.205 & $0.86(0.48-1.54)$ & 0.604 \\
\hline \multicolumn{5}{|l|}{ Residual disease } \\
\hline$<1 \mathrm{~cm}$ (Optimal debulking) & Reference & & & \\
\hline$\geq 1 \mathrm{~cm}$ (Suboptimal debulking) & $3.1(2.5-3.9)$ & $<0.001$ & $2.47(1.92-3.19)$ & $<0.001$ \\
\hline
\end{tabular}

$H R$ hazard ratio, $C /$ confidence interval, $N L R$ neutrophil to lymphocyte ratio, FIGO International Federation of Gynecology and Obstetrics.

* Adjusted all the other factors. 


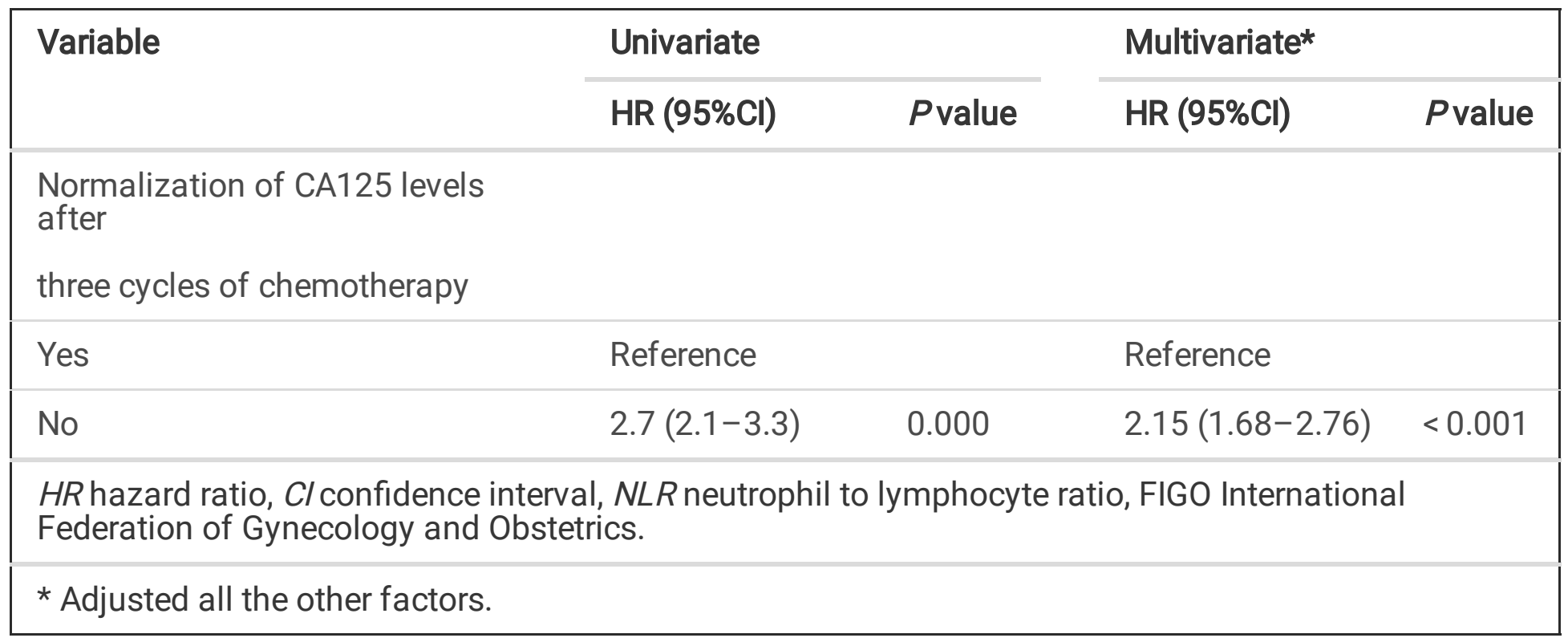

Development of the Nomogram for PFS Prediction

A nomogram for PFS prediction that incorporated the independent predictors indicated by univariate analysis was developed in the primary cohort (Fig. 1 and Appendix 2). In the nomogram, FIGO stage made the greatest contribution to PFS prediction, followed by residual disease status and the normalization of CA125 levels after three cycles of chemotherapy. A certain score was assigned to each variable. By summing the scores from all the variables and locating the scores on the total point scale, the probabilities of 1-year PFS and 3-year PFS could be determined by drawing a vertical line from the total points' axis.

Performance of the Nomogram in the Primary Cohort

Internal validation was performed using bootstrapping resampling. After 1000 repetitions, the bootstrapcorrected C-index for the nomogram was 0.708 (95\% confidence interval [Cl], 0.674 to 0.741 ). The calibration plots of the nomogram revealed optimal agreement between the predicted probability and the actual 1-year PFS and 3-year PFS rates in the primary cohort (Fig. 2A and B).

Validation of the Nomogram

When applied to the external validation cohort, the nomogram achieved an excellent C-index of 0.724 $(95 \% \mathrm{Cl}, 0.649$ to 0.798$)$ for PFS prediction. The calibration plots showed good consistency between the nomogram-predicted and observed 1- and 3-year PFS in the external validation cohort (Fig. 2C and D).

Incremental Predictive Value of CA125 Levels to the Nomogram

The incremental predictive value of CA125 levels after three cycles of chemotherapy to the nomogram was evaluated by the comparison of the C-index for the models with and without CA125 levels. CA125 levels after three cycles of chemotherapy showed a marginally significant increment of discrimination to the nomogram in the primary cohort ( $C$-index, 0.708 vs $0.668 ; \mathrm{P}=0.097$ ) 
First, we compared the performance of the nomogram and the FIGO staging system with respect to the Cindex. In the primary cohort, a higher $\mathrm{C}$-index was achieved for the nomogram compared with the FIGO staging system only (C-index, 0.708 vs $0.578 \mathrm{P}<0.001)$.

Next, we calculated a total score for each patient by adding the individual points for each risk factor presented by the nomogram. Then, we classified the patients into low, moderate, and high risk groups by ranking their scores calculated by the nomogram into tertiles; each group represented a distinct prognosis. The PFS curves for these risk groups, as well as for different FIGO stages, are presented in Fig. 3. Although both the FIGO staging system and the nomogram could stratify patients with distinct prognoses, the Kaplan-Meier survival curves implied that the nomogram could more effectively stratify patients with distinct PFS.

Clinical application

DCA was applied to the nomograms with and without integration of CA125 levels after three cycles of chemotherapy (Fig. 4). The decision curve for the model incorporating CA125 levels showed that if the threshold probability was greater than $15 \%$, the nomogram for PFS prediction had more benefits than the treat-none and treat-all schemes. Moreover, the nomogram integrating CA125 levels after three cycles of chemotherapy had a superior net benefit over the model that did not include CA125 levels for a wide range of threshold probabilities.

\section{Discussion}

We constructed and validated a nomogram for predicting PFS in patients with ovarian cancer. The nomogram incorporated the novel variable of the normalization of CA125 levels after three cycles of chemotherapy, as well as other clinical risk factors. Normalization of CA125 levels after three chemotherapy cycles has been demonstrated as an important prognostic factor for patients with ovarian cancer. Integration of this variable to the nomogram facilitated individualized PFS prediction for ovarian cancer.

A series of prognostic nomograms have been developed for the prediction of ovarian cancer survival. However, most of these models are based on variables reflecting pathological characteristics (e.g. tumor grade and histology) and prognostic information (e.g. age, residual disease status, and preoperative albumin level), and achieved C-indices ranging from 0.63 to $0.72 .[7,8,19,20]$ Clark et al.[21] developed a nine-variable prognostic nomogram that presented an excellent $C$-index of 0.727 for advanced stage ovarian cancer. However, that model was not validated, nor did it have widespread clinical utility. The lack of applicability may be attributed to the incorporation of a rarely used residual disease grouping $(<2 \mathrm{~cm}$, 2-5 $\mathrm{cm}$, or $>5 \mathrm{~cm}$ ) and too many variables in the model. Thus far, few nomograms have been constructed with the integration of factors reflecting treatment effect. Serum CA125 is a widely used biomarker in ovarian cancer that has been applied to monitor the response of chemotherapy for a long 
time.[11-16] In the current study, we developed a nomogram for PFS prediction for ovarian cancer by integrating the normalization of CA125 levels after three chemotherapy cycles and other risk factors, which demonstrated adequate discrimination in the primary cohort (C-index, 0.708). The nomogram was further validated by an independent external validation cohort to avoid overfitting and determine the generalizability. Surprisingly, an improved C-index of 0.724 was obtained in the external validation cohort, which implied that our nomogram was robust for prediction of PFS. In this study, calibration plots showed good consistency between prediction and actual observation, which ensured the reliability and repeatability of our nomogram.

To the best of our knowledge, this is the first nomogram to incorporate the variable of normalization of CA125 levels after three chemotherapy cycles. Integration of this variable into the nomogram improved the discriminative ability when compared with the prediction model that did not include CA125 levels (Cindex, 0.708 vs $0.668 ; 0.04$ difference). Although the statistics did not reach the conventional significance, we believe that this was because of the small sample size.

FIGO stage is a well-established prognostic factor for ovarian cancer.[22, 23] Nevertheless, insufficiency in outcome prediction still remains in the FIGO staging system. In the current study, superior discriminatory ability was observed for the established nomogram as compared to the FIGO staging system. Moreover, the Kaplan-Meier curves for risk grouping based on the established nomogram successfully stratified patients with distinct prognoses in the primary cohort. This implied that our prognostic nomogram could be better able to stratify patients into different risk subgroups, which might be helpful to guide optimal individual treatment decision-making.

In addition, to assess the clinical value of the nomogram, DCA was performed. The decision curve showed that if the threshold probability of a patient or doctor is greater than $15 \%$, using the nomogram in the current study for PFS prediction in ovarian cancer added more benefit than either the treat-none or treat-all schemes. Furthermore, DCA revealed that the nomogram incorporating normalization of CA125 levels after three chemotherapy cycles had a superior net benefit over the model that did not include CA125 levels, for a wide range of threshold probabilities.

One strength of our nomogram was that all the variables in the model were generated as part of routine practice, and therefore did not need further classification and definition. This facilitated easy use of our nomogram in clinical practice. In this study, the variable of tumor grade was not significantly associated with PFS in univariate and multivariate analyses. Considering that tumor grade is an important prognostic factor in ovarian cancer,[24, 25] we also employed it to develop the nomogram. We believe that the main reason for its insignificance was that the majority of patients $(81.7 \%)$ presented with grade 3 tumors. In addition, we also tested a model of the nomogram without the variable of tumor grade, and the discrimination ability was only slightly impaired (C-index, 0.702).

However, our study has several limitations. First, it was reported that approximately $7.6 \%$ of the patients with advanced ovarian cancer had normal CA125 levels prior to treatment.[26, 11] For these patients, a decline in CA125 levels during adjuvant chemotherapy may not be a good prognostic predictor. Second, 
the number of patients in the external validation cohort was small. Finally, our nomogram is limited by the retrospective nature of the data collection.

\section{Conclusions}

In conclusion, we developed and validated a nomogram incorporating the normalization of CA125 levels after three chemotherapy cycles for PFS prediction in patients with ovarian cancer. This nomogram showed good predictive performance and easy clinical application. It may help guide optimal treatment decision-making. Further external evaluations using other independent cohorts are warranted.

\section{Abbreviations}

CA125: Cancer antigen 125; PFS: Progression-free survival; OS: Overall survival; C-index: Concordance index; FIGO: International Federation of Gynecology and Obstetrics; CT: Computed tomography; MRI: Magnetic resonance imaging; PET-CT: Positron emission tomography- computed tomography; DCA: Decision curve analysis; $\mathrm{Cl}$ : Confidence interval.

\section{Declarations}

\section{Acknowledgements}

We would like to thank our tutor Chunyan Lan for her careful guidance.

\section{Authors' contributions}

All authors contributed to the study conception and design. Material preparation, data collection and analysis were performed by ZL, QG. The first draft of the manuscript was written by MH, HP and all authors commented on previous versions of the manuscript. All authors have read and approved the final manuscript.

\section{Funding}

No funding.

\section{Availability of data and materials}

The datasets used and/or analyzed during the current study are available from the corresponding author on reasonable request.

\section{Ethics approval and consent to participate}

Ethical approval was obtained from the institutional review board of Sun Yat-sen University Cancer Centre. The need for informed consent was waived since no identifiable information was used in this study. 


\section{Consent for publication}

All authors agree to publish the paper.

\section{Competing interests}

The authors declare that they have no competing interests.

\section{References}

1. Siegel RL, Miller KD, Jemal A. Cancer statistics. 2018. CA Cancer J Clin.2018; 68 (1):7-30.

2. Chen W, Zheng R, Baade PD, et al. Cancer statistics in China, 2015. CA Cancer J Clin. 2016;66(2):115-32.

3. du Bois A, Luck HJ, Meier W, et al. A randomized clinical trial of cisplatin/paclitaxel versus carboplatin/paclitaxel as first-line treatment of ovarian cancer. J Natl Cancer Inst. 2003;95(17):1320-29.

4. Piccart MJ, Bertelsen $\mathrm{K}$, James $\mathrm{K}$, et al. Randomized intergroup trial of cisplatin-paclitaxel versus cisplatin-cyclophosphamide in women with advanced epithelial ovarian cancer: three-year results. $J$ Natl Cancer Inst. 2000;92(9):699-708.

5. Iasonos A, Schrag D, Raj GV, et al. How to build and interpret a nomogram for cancer prognosis. J Clin Oncol. 2008;26(8):1364-70.

6. Chi DS, Palayekar MJ, Sonoda Y, et al. Nomogram for survival after primary surgery for bulky stage IIIC ovarian carcinoma. Gynecol Oncol. 2008;108(1):191-4.

7. Barlin JN, Yu C, Hill EK, et al. Nomogram for predicting 5-year disease-specific mortality after primary surgery for epithelial ovarian cancer. Gynecol Oncol. 2012;125(1):25-30.

8. Lee CK, Simes RJ, Brown C, et al. A prognostic nomogram to predict overall survival in patients with platinum-sensitive recurrent ovarian cancer. Ann Oncol. 2013;24(4):937-43.

9. Bast RC Jr, Klug TL, St John E, et al. A radioimmunoassay using a monoclonal antibody to monitor the course of epithelial ovarian cancer. N Engl J Med. 1983;309(15):883-7.

10. Jacobs I, Bast RC. Jr. The CA 125 tumour-associated antigen: a review of the literature. Hum Reprod. 1989;4(1):1-12.

11. Lee M, Chang MY, Yoo H, et al. Clinical Significance of CA125 Level after the First Cycle of Chemotherapy on Survival of Patients with Advanced Ovarian Cancer. Yonsei Med J. 2016;57(3):580-7.

12. Markman M, Federico M, Liu PY, et al. Significance of early changes in the serum CA-125 antigen level on overall survival in advanced ovarian cancer. Gynecol Oncol. 2006;103(1):195-8.

13. Rocconi RP, Matthews KS, Kemper MK, et al. The timing of normalization of CA-125 levels during primary chemotherapy is predictive of survival in patients with epithelial ovarian cancer. Gynecol Oncol. 2009;114(2):242-5. 
14. Lan CY, Huang H, Liu JH. [Prognostic value of serum CA(125) level change during chemotherapy post-surgery in patients with advanced epithelial ovarian carcinoma]. Zhonghua Fu Chan Ke Za Zhi. 2008;43(10):732-6.

15. Skaznik-Wikiel ME, Sukumvanich P, Beriwal S, et al. Possible use of CA-125 level normalization after the third chemotherapy cycle in deciding on chemotherapy regimen in patients with epithelial ovarian cancer: brief report. Int J Gynecol Cancer. 2011;21(6):1013-7.

16. Tian $\mathrm{C}$, Markman M, Zaino $\mathrm{R}$, et al. CA-125 change after chemotherapy in prediction of treatment outcome among advanced mucinous and clear cell epithelial ovarian cancers: a Gynecologic Oncology Group study. Cancer. 2009;115(7):1395-403.

17. Vickers AJ, Elkin EB. Decision curve analysis: a novel method for evaluating prediction models. Med Decis Making. 2006;26(6):565-74.

18. Vickers AJ, Cronin AM, Elkin EB, et al. Extensions to decision curve analysis, a novel method for evaluating diagnostic tests, prediction models and molecular markers. BMC Med Inform Decis Mak. 2008;8:53.

19. Gerestein CG, Eijkemans MJ, de Jong D, et al. The prediction of progression-free and overall survival in women with an advanced stage of epithelial ovarian carcinoma. BJOG. 2009;116(3):372-80.

20. Wahner Hendrickson AE, Hawthorne KM, Goode EL, et al. Assessment of published models and prognostic variables in epithelial ovarian cancer at Mayo Clinic. Gynecol Oncol. 2015;137(1):77-85.

21. Clark TG, Stewart ME, Altman DG, et al. A prognostic model for ovarian cancer. Br J Cancer. 2001;85(7):944-52.

22. Bristow RE, Tomacruz RS, Armstrong DK, et al. Survival effect of maximal cytoreductive surgery for advanced ovarian carcinoma during the platinum era: a meta-analysis. J Clin Oncol. 2002;20(5):1248-59.

23. du Bois A, Reuss A, Pujade-Lauraine E, et al. Role of surgical outcome as prognostic factor in advanced epithelial ovarian cancer: a combined exploratory analysis of 3 prospectively randomized phase 3 multicenter trials: by the Arbeitsgemeinschaft Gynaekologische Onkologie Studiengruppe Ovarialkarzinom (AGO-OVAR) and the Groupe d'Investigateurs Nationaux Pour les Etudes des Cancers de l'Ovaire. (GINECO)Cancer. 2009;115(6):1234-44.

24. Aletti GD, Dowdy SC, Podratz KC, et al. Relationship among surgical complexity, short-term morbidity, and overall survival in primary surgery for advanced ovarian cancer. Am J Obstet Gynecol. 2007;197(6):676 e671-7.

25. Goode EL, Maurer MJ, Sellers TA, et al. Inherited determinants of ovarian cancer survival. Clin Cancer Res. 2010;16(3):995-1007.

26. Paramasivam S, Tripcony L, Crandon A, et al. Prognostic importance of preoperative CA-125 in International Federation of Gynecology and Obstetrics stage I epithelial ovarian cancer: an Australian multicenter study. J Clin Oncol. 2005;23(25):5938-42.

\section{Figures}


Points

Residual disease

FIGO Stage

Grade

CA125 levels after three cycles of chemotherapy

Age

Total Points

1-year PFS

3-year PFS

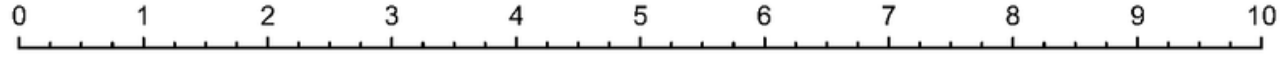

$\geq 1 \mathrm{~cm}$ (Suboptimal debulking)

$$
<1 \mathrm{~cm} \text { (Optimal debulking) }
$$

\begin{tabular}{llll}
\hline & & Stage III & Stage IV \\
& & & \\
& & & \\
\hline Stage II & Grade3 & &
\end{tabular}

High

Normal

$>50$ years

$<=50$ years
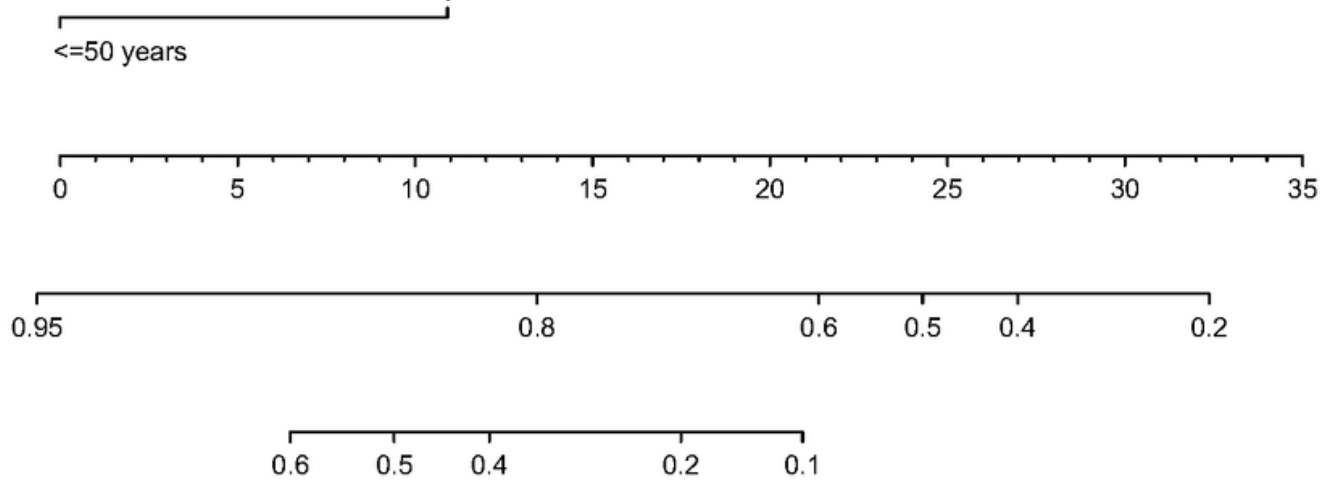

\section{Figure 1}

Nomogram incorporating cancer antigen 125 levels after three cycles of chemotherapy for the prediction of progression-free survival in patients with ovarian cancer in the primary cohort. 
A

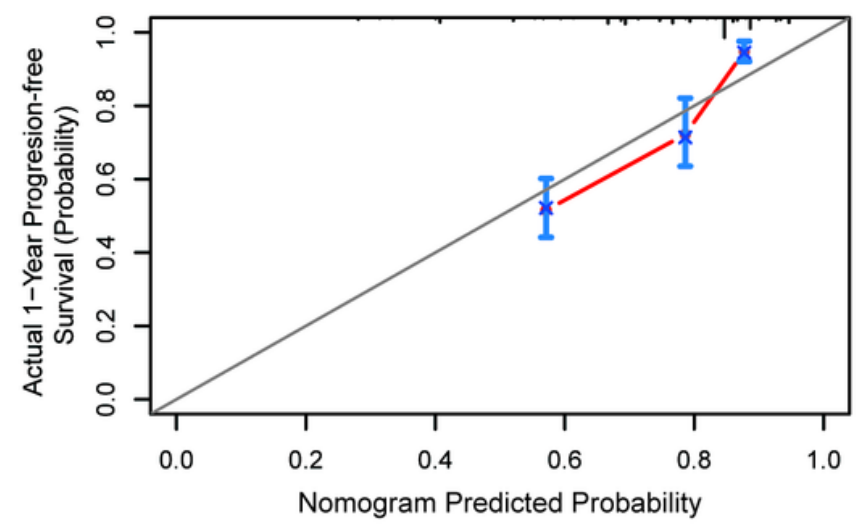

C

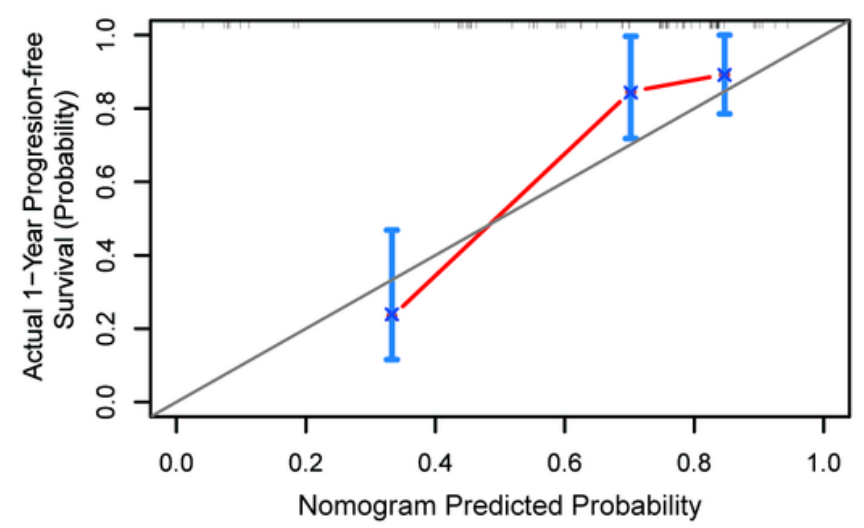

B

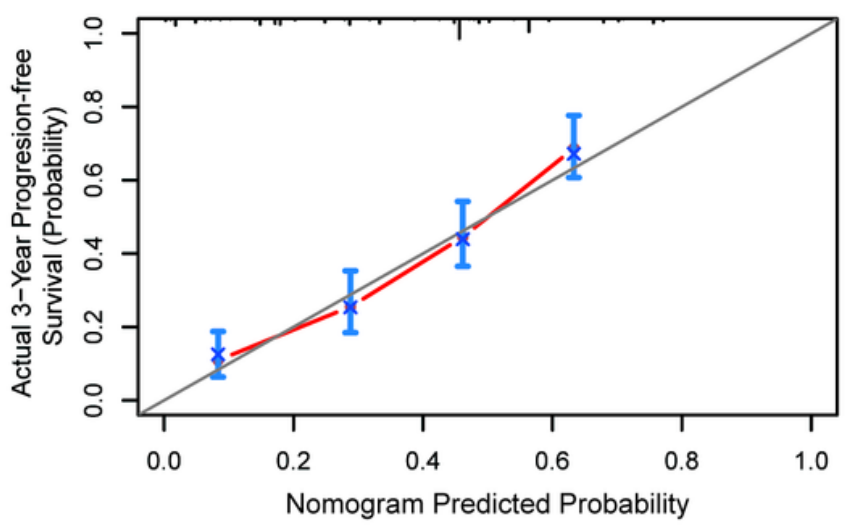

D

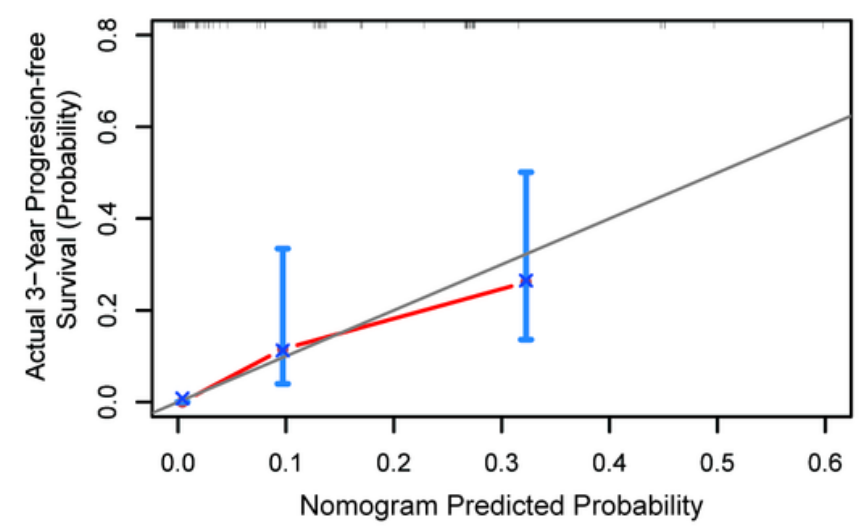

\section{Figure 2}

The calibration plots of the established nomogram for predicting progression-free survival. Calibration plots for predicting (A) 1-year and (B) 3-year progression-free survival in the primary cohort. Calibration plots for predicting (C) 1-year and (D) 3-year progression-free survival in external validation cohort. The yaxis represents the actual progression-free survival rates. The $x$-axis represents the predicted progressionfree survival rates. 

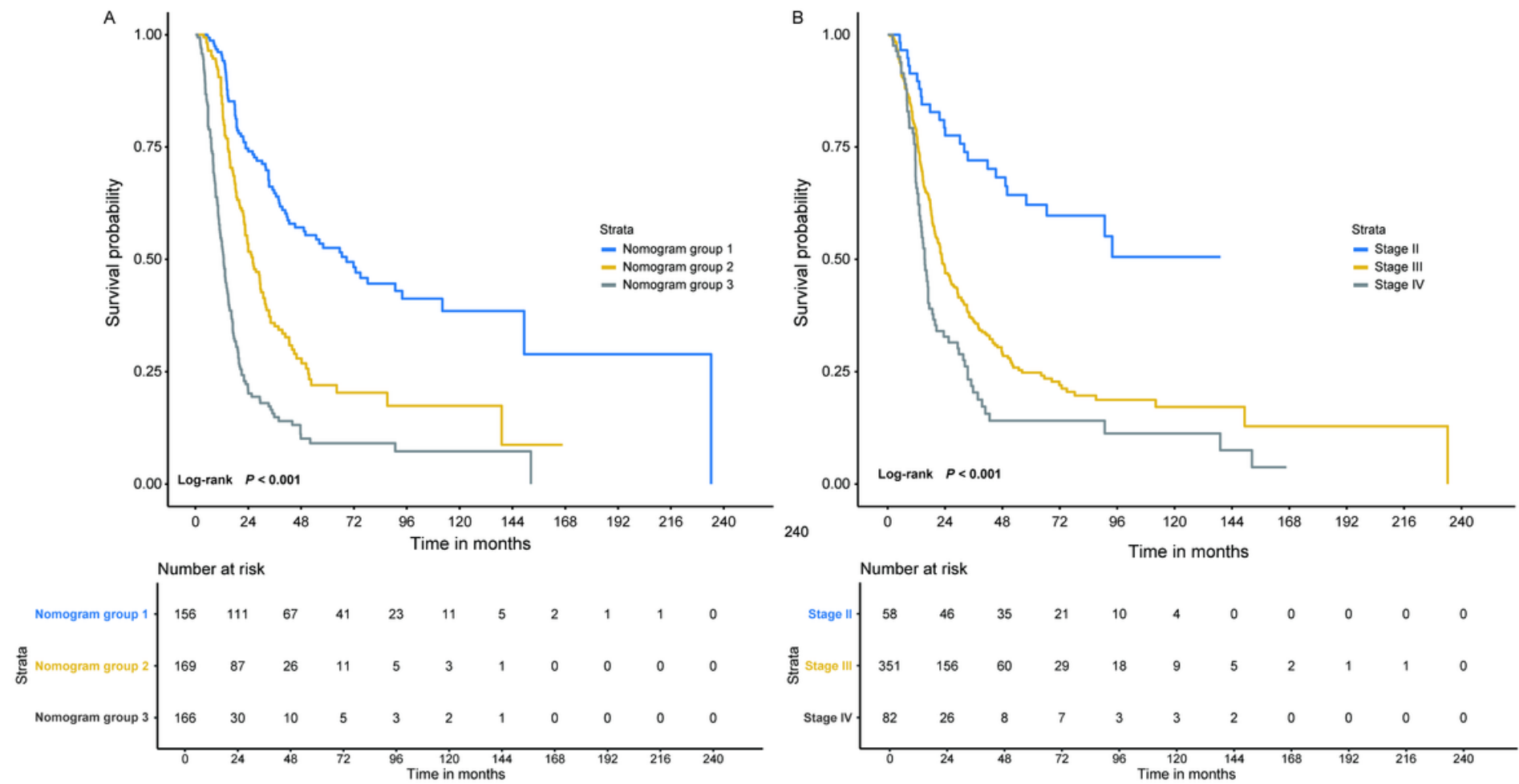

\section{Figure 3}

Kaplan-Meier survival curves for patients in the primary cohort stratified by scores calculated by $(A)$ the established nomogram and (B) the International Federation of Gynecology and Obstetrics staging system. 


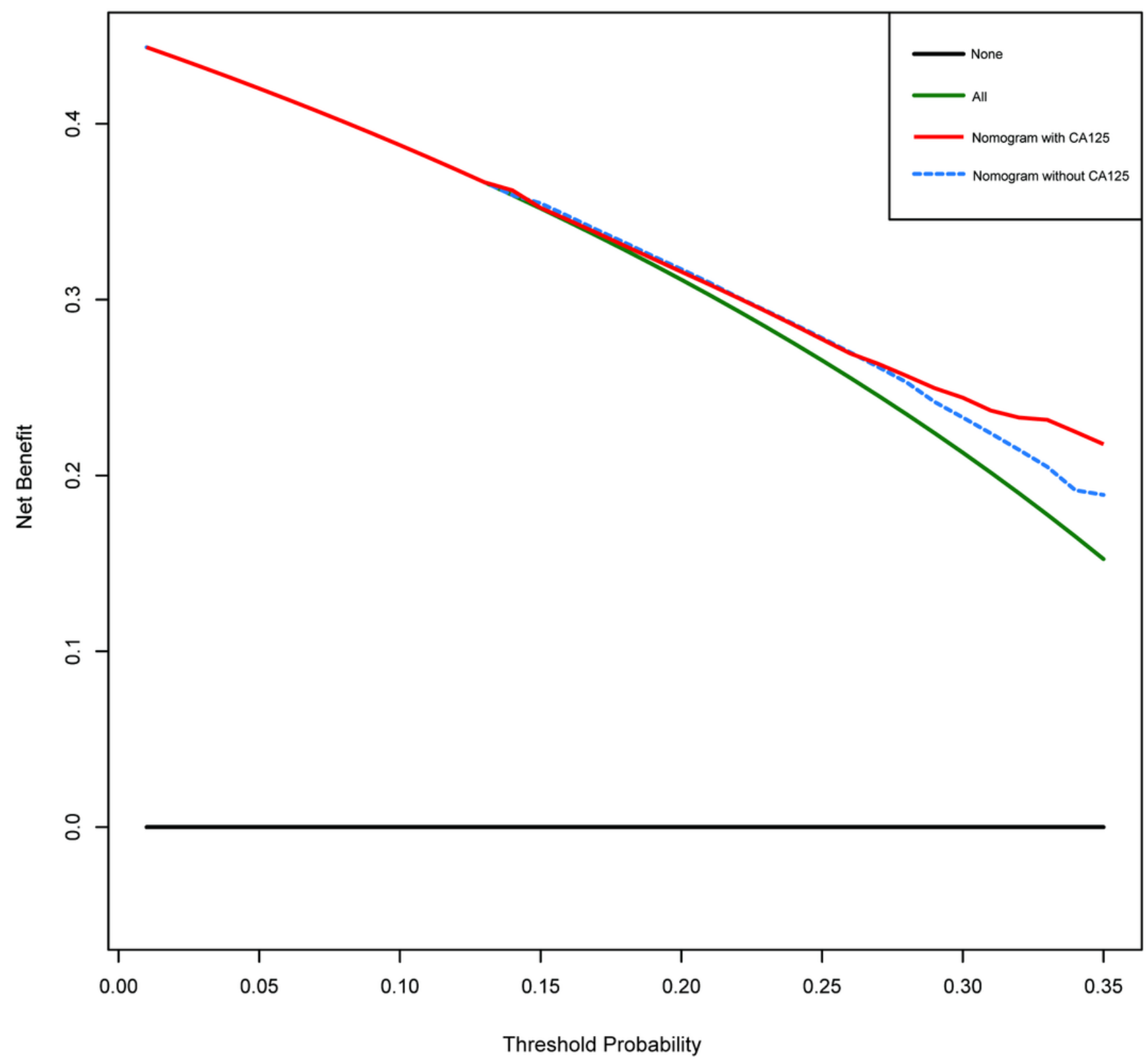

\section{Figure 4}

Decision curve analysis for the nomograms with and without integration of cancer antigen 125 (CA125) levels after three cycles of chemotherapy to predict progression-free survival in ovarian cancer. The $y$-axis measures the net benefit. The green line represents the assumption that all patients experienced recurrence. The black line represents the assumption that no patients experienced recurrence. The red line represents the model incorporating CA125 levels after three cycles of chemotherapy. The blue dotted line represents the model without CA125 levels after three cycles of chemotherapy. The nomogram with integration of CA125 levels after three cycles of chemotherapy showed superior clinical net benefit over the model without CA125 levels. 


\section{Supplementary Files}

This is a list of supplementary files associated with this preprint. Click to download.

- Additionalfile1.docx

- Additionalfile2.docx 